\section{ScienceDirect}

BIOETHICS UPdate 6 (2020) 101-114
BIOETHICS UPdate

\title{
De la sociedad maquinal a la sociedad de la vida y la biósfera
}

From the machine society to the life and biosphere society

\author{
Luis Felipe Abreu-Hernández \\ Profesor de la División de Estudios de Posgrado, Facultad de Medicina, Universidad Nacional \\ Autónoma de México, Coyoacán, Ciudad de México, México \\ Recibido el 10 de septiembre de 2020; aceptado el 20 de septiembre de 2020 \\ Disponible en Internet el 29 de septiembre de 2020
}

\section{Resumen}

Estamos ubicados en una inmensa crisis social y ecológica, de la cual el calentamiento global y la pandemia de la COVID-19 son una muestra. Nuestros sistemas productivos y sociales han sido modelados sobre la base de la mecánica y mediante respuestas lineales que han convertido a los humanos en seres instrumentales empleados en tareas rutinarias. Además, la búsqueda de resultados inmediatos ha generado la crisis ecológica. Nuestro futuro como especie depende de nuestra capacidad de aprender las lecciones de la vida y los ecosistemas, con el fin de abrir nuevos horizontes y generar nuevas geometrías que nos permitan co-evolucionar con la biósfera. La tarea es inmensa porque nos confrontamos con la frontera de lo muy complejo, de lo entretejido e interconectado, de lo dinámico, y no acertamos a visualizar que la suma de las

Correos electrónicos: luisabreu03@yahoo.com, lfah@unam.mx 
ganancias individuales no es una ganancia social, y mucho menos para la biósfera. La bioética resulta central para enlazar lo humano con la vida y la biósfera.

(C) 2020 Publicado por Masson Doyma México S.A. en nombre de Centros Culturales de México, A.C.

Palabras clave: COVID-19; Mecánica; Bioética; Bioesfera

Abstract: We are situated in an immense social and ecological crisis, of which global warming and the COVID-19 pandemic are examples. Our productive and social systems have been modelled on the basis of mechanics and linear responses that have turned humans into instrumental beings employed in routine tasks. Furthermore, the search for immediate results has generated the ecological crisis. Our future as a species depends on our ability to learn the lessons of life and ecosystems, in order to open new horizons and generate new geometries that allow us to co-evolve with the biosphere. The task is immense because we are confronted with the frontier of the very complex, of the interwoven and interconnected, of the dynamic, and we fail to visualize that the sum of individual gains is not a social gain, and much less for the biosphere. Bioethics is central to linking the human with life and the biosphere.

(C) 2020 Published by Masson Doyma México S.A. on behalf of Centros Culturales de México, A.C.

Keywords: COVID-19; Mechanics; Bioethics; Biosphere

\section{El mecanicismo social}

El modelo social derivado de la revolución industrial ha puesto en el centro la producción de máquinas, que son artificios que utilizan energía para dirigirla al logro de los propósitos humanos. Las máquinas nos permitieron multiplicar nuestra fuerza, con el fin de incidir sobre la naturaleza modificándola para obtener éxitos puntuales, que han sido el centro de nuestra sociedad que está llegando a su fin en medio de una crisis ecológica global. No implica esto que debamos renunciar a poseer máquinas para retornar a una vida pastoril y bucólica, sino que la época del mecanicismo social ha llegado a sus límites, y que la lógica que ordena nuestras vidas ya no debe ser la simplicidad maquinal, sino la lógica de lo viviente, con su diversidad, su adaptabilidad y capacidad autoorganizativa; hoy debemos generar una nueva visión ecosistémica para contender con la crisis global más grave en muchas décadas.

La modernidad se sustentó en crear máquinas predecibles que deben garantizar ciertos resultados con precisión y regularidad, por lo cual están organizadas bajo los 
principios de la invariancia y la estabilidad (Polanyi, 1968). No obstante, la producción de máquinas invariantes es paradójicamente resultado de la variabilidad humana y de nuestra capacidad imaginativa. Surge así lo que podemos denominar la contradicción básica de la modernidad, la confrontación entre la rigidez de las máquinas y la variabilidad humana, o si se quiere llevar hasta sus últimas consecuencias: la confrontación entre la vida y el mecanicismo social lineal y brutal frente al cual la vida resulta irreducible.

Las máquinas son replicadas por los procesos industriales cientos de miles o millones de veces, y para ello hemos generado métodos bien establecidos, como el denominado «sistema de partes intercambiables» que permite que unas piezas encajen con otras de manera precisa poniendo «límites de tolerancia» que establecen y garantizan bajo diseño las dimensiones aceptables, establecidas para facilitar el ensamblado o la reparación de las máquinas. Surge con ello la estandarización de procesos y el denominado control de la calidad, así como una sociedad normada para replicar estos procesos de manera estandarizada.

\section{El uso instrumental de los seres humanos}

La fabricación de piezas y el ensamblado de las máquinas han sido actividades humanas esenciales, y nos encontramos con un conflicto directo entre las exigencias de procesos estandarizados que buscan eliminar toda fuente de variación y la variabilidad y creatividad de los seres humanos que debe ser severamente limitada porque se convierte en fuente de errores o acciones descoordinadas. Las acciones humanas son descompuestas en sus elementos molares, controladas y transformadas en rutinas simples e invariantes operadas frecuentemente al margen de la consciencia. La realización de rutinas segmentadas permitió que grandes multitudes con baja preparación pudiesen habilitarse en semanas para incorporarse a la producción. Finalmente, el fordismo inventó la línea de montaje al colocar una banda transportadora que impone el ritmo de trabajo a los humanos subordinándolos a la máquina, y con ello grandes ejércitos industriales marcharon al ritmo impuesto por un inmenso mecanismo industrial (Coriat, 2008). La variabilidad humana se diluyó aún más para millones de trabajadores, que fueron capacitados para seguir instructivos y repetir tareas; su variabilidad y creatividad se limitó para acoplarlos a la cadencia de las máquinas y se les convirtió en lo que Riesman ha denominado «other directed persons» (Riesman, Glazer y Denney, 2001). Esta clase de organización social era en sí misma una verdadera máquina social para producir máquinas y productos. La producción fabril es un verdadero sistema de amplificación en el cual una minoría dirige, diseña y 
concentra la capacidad creativa y directiva, y la gran mayoría reproduce lo diseñado, sigue instrucciones, aplica rutinas y posee una capacidad limitada para pensar por sí misma.

El poder social se construyó en torno del control de la «producción de los medios de producción», y por supuesto incluyó también la fabricación de los medios de destrucción o armamento, indispensable para defender los bastiones de los Estados nacionales y avanzar en la conquista de territorios coloniales, transformados en enclaves para la obtención de materias primas y mano de obra cuasi esclava de baja productividad, pero que adquiere valor económico al reducir sus niveles de consumo por debajo del valor de lo producido y al aumentar su volumen mediante el uso de millones de seres empobrecidos y violentados por los ejércitos colonialistas. La lucha por territorios coloniales, por el control de las materias primas y los mercados desató confrontaciones por el reparto del mundo y desembocó en dos guerras mundiales.

\section{La visión lineal y la destrucción de la biósfera}

La visión reduccionista de la realidad como producto de la suma de relaciones causaefecto crea un universo lineal y aditivo inexistente. En la base de este paradigma mecánico se encuentra la noción de causalidad con sus tres supuestos principales: 1 . Precedencia temporal: que establece la necesaria anticipación de la causa frente al efecto. 2. Covariación: que expresa que el efecto se produce en función de la causa y presenta una relación homogénea y lineal, cuya tangente permanece inalterable al cambio de magnitud; y 3. Aislamiento: el cual implica que no exista otra causa adicional capaz de explicar el efecto, o en caso de existir debe ser eliminada o controlada. Precisamente los diseños experimentales se han desarrollado para aislar los fenómenos y eliminar los llamados factores de confusión. Este último principio es el más perverso de todos, pues al aislar los fenómenos transforma a los sistemas abiertos, múltiplemente conectados y complejos, en ambientes cerrados, unicausales, lineales, acotados y predecibles y con ello desaparece la magia de lo viviente y autoorganizado. Estas cadenas causales unidireccionales simplifican la naturaleza, porque se diseñan experimentos acotados a un número mínimo de variables, se eliminan todos aquellos elementos que pudiesen perturbar el experimento, como la causalidad recíproca, las gasas de realimentación y toda complejidad; en esta condición artificiosa la linealidad es una profecía autocumplida. Así la vida, a la cual pertenece lo humano, queda convertida en una anomalía. Hay que ver la dificultad de las ciencias naturales para intentar comprender la vida, y debemos revalorar el esfuerzo monumental de Schrödinger para lograr que la ciencia aceptara la vida como 
un fenómeno posible resultado de la entropía negativa y la autoorganización de los sistemas biológicos (Schrödinger y Penrose, 1992), y que a la postre impulsaría los estudios de difracción de rayos X que permitirían el descubrimiento del ADN.

Pero retornemos al análisis sobre la influencia del mecanicismo en la sociedad humana. Cuando construimos máquinas que poseen su fuente de energía y nos sirven para influir y modificar la naturaleza de forma unidireccional, dichas máquinas establecen una mediación entre nosotros y la naturaleza, así dejamos de confrontarnos con ella de manera directa y ya no la percibimos en su complejidad (Vetlesen, 2012), de tal manera que podemos enfocarnos centralmente en lograr nuestro propósito, despreciando y externalizando otros efectos. Por este camino la poderosa linealidad motorizada destruye los ecosistemas autoorganizados. La máquina aparenta ser una prolongación de nosotros, pero en ese proceso más bien nosotros nos hemos transformado en una prolongación de la máquina, y terminamos asumiéndonos como una máquina más, perdiendo con ello nuestro carácter humano, desapareciendo toda generosidad y visión de largo plazo. Por este camino, puedo deforestar una zona de la selva tropical protegido en una cabina de un gran tractor de orugas con aire acondicionado y música de fondo, sin percatarme de los gritos desesperados de los monos, de los nidos con crías que caen, o del venado que huye del hábitat que estoy destruyendo. Como siempre esta situación brutalmente mecánica se manifiesta con mayor claridad en la guerra y en las máquinas para matar, y así a miles de kilómetros de distancia, ubicados cómodamente en un cuartel con aire acondicionado y con un café recién hecho, sentados frente a una computadora están personas que pueden operar drones militares, sintiéndose empoderadas y seguras para disparar balas o misiles, sin mirar los ojos de quienes están eliminando, y todavía dicen los expertos que esto reduce el «síndrome de desgaste por empatía», la cual se produce solo cuando eres capaz de sentir el dolor del otro. Así por este camino se elimina la empatía, y el ser humano cooperativo, el que posee «neuronas espejo» para sentir al otro (Rifkin, 2010), es substituido por un ser humano mecánico, insensible y brutal. Bien afirma Hanna Arendt que el totalitarismo está interconstruido en la sociedad moderna y, como podemos apreciar, es producto directo de la deshumanización (Arendt, 1994).

La sociedad mecánica basada en la visión de la causalidad lineal y homogénea típica de la modernidad se configura por una multiplicidad de seres humanos enfocados en controlar causas eficientes para obtener resultados inmediatos, determinados por la búsqueda de beneficios y apoyados en un sistema social que reduce la cooperación, pero que han sido interiorizados y asumidos por las personas como propios, los cuales son presentados como libertad, competencia, éxito o simplemente sobrevida 
del más fuerte. En consecuencia, la interacción con otros seres humanos se ve deformada porque actuamos sobre la base de competir para obtener ventaja de los otros, y en general los miro a todos como competidores. Por necesidad me vuelvo desconfiado porque asumo que también intentarán obtener ventaja sobre mí. Esta condición se expresa como un individualismo extremo y un gran aislamiento, y desemboca en la soledad típica de la modernidad y de las sociedades llamadas «avanzadas».

Pero no solo los seres humanos individuales son poseídos por la máquina que supuestamente manejan; también las grandes corporaciones operan como gigantescas maquinarias orientadas a lograr sus propósitos y metas particulares por encima de la sociedad, la ecología y la biósfera, justifican la manipulación de precios y mercados, y se constituyen en organizaciones impersonales orientadas a maximizar sus ganancias pasando por encima de las personas, los ecosistemas y la vida. Milton Friedman expresó este punto de vista cuando escribió un artículo denominado: «La responsabilidad social de la empresa es incrementar sus ganancias» en el cual afirmaba:

«La doctrina de la «responsabilidad social» involucra aceptar el punto de vista socialista, que los mecanismos políticos y no los mecanismos de mercado, son la manera apropiada para determinar la asignación de recursos escasos para usos alternativos» (Friedman, 1970).

Friedman sostiene que la máquina social del mercado se encarga de hacerlo de manera automática al incrementar las ganancias monetarias.

Esta posición sería válida solo si aceptamos que la organización social es reduccionista, y lo que es bueno para una empresa «X», por ese solo hecho es valioso para la sociedad en su conjunto. En el caso extremo se podría afirmar «Si produce ganancias es bueno», y de esa forma deberíamos permitir el narcotráfico, el comercio de personas, el tráfico de armas, entre otras cosas. El mercado tiene un papel relevante en la autorregulación social, pero cuando este falla se debe intervenir, legislar y acotar las actividades perniciosas, que generan ganancias y que simultáneamente destruyen a la sociedad y le restan valor social.

Un ejemplo de esta clase de reduccionismo ha sido el avión 737 Max de Boeing, investigado por un comité de la Cámara de Diputados Norteamericana que concluyó que: «Boeing emphasized profits over safety» («Boeing enfatizó las ganancias sobre la seguridad») (Chokshi, 2020). 
Durante el siglo pasado se pretendió que el socialismo sería una alternativa al capitalismo, cuando en realidad es otra variante del mecanicismo social. El propio Lenin afirmó que el socialismo debería fundarse también en la «administración científica de Taylor», y que el trabajo debía «militarizarse», poniendo al frente de esta labor a Trotsky (Merkle, 1980), con lo cual contribuyó a fortalecer un sistema totalitario con Stalin al frente. El socialismo y su ideal de lograr una planificación centralizada de la economía solo se podía alcanzar a plenitud eliminando o restringiendo la innovación, porque solamente es posible la planificación centralizada perfecta si podemos conocer el futuro y eliminamos las innovaciones que generan cambios impredecibles y producen una destrucción creativa. Únicamente de esa manera se puede lograr una mecanización burocrática perfecta y construir una inmensa máquina donde todo opera conforme a lo planeado, pero ello tiende a eliminar cualquier proceso de autoorganización e innovación, destruye la creatividad y las redes sociales transformadoras que pudiesen retar al poder centralizado.

La confrontación entre estos dos supuestos sistemas sociales «diferentes» dio origen a la Guerra Fría y obligó a la Unión Soviética a instrumentar un proceso de innovación en el terreno militar montado sobre un sistema incapaz de favorecer la autoorganización productiva de la sociedad, lo cual terminó por demostrar su ineptitud para construir una economía fuerte y en expansión constante que pudiese soportar el esfuerzo militar.

De otra parte, los países capitalistas se vieron obligados a desarrollar sistemas de ciencia y tecnología vinculados a mantener un poder militar y político, y debieron constituir un «estado de bienestar» capaz de asegurar que las grandes masas les fuesen leales en caso de una tercera guerra mundial, a la vez que mantenían un fuerte control político y policiaco para evitar perder el control de sus territorios.

Uno de los logros colaterales de este periodo fue el desarrollo de la ciencia y la ingeniería, que dio origen a la industria aeroespacial, la microelectrónica, las computadoras personales, las telecomunicaciones, la informática, Internet, la robótica y la inteligencia artificial, y estas a su vez fueron la base para el desarrollo del proyecto del genoma humano y la nueva biología.

Debemos destacar que la robótica, la automatización de procesos y el desarrollo de los sistemas flexibles de producción aunados a la inteligencia artificial han permitido automatizar crecientemente la producción, y ahora es posible incrementar la producción con una franca reducción del trabajo humano. 


\section{La automatización del mecanicismo social}

La búsqueda de la ganancia también se ha automatizado, y las computadoras y sistemas de inteligencia artificial han sido programados para realizar maniobras especulativas capaces de generar ganancias día y noche, sin intervención humana y sin ninguna consideración ética. Estas máquinas pueden colapsar el valor de las materias primas y desolar regiones enteras o quebrar países sin ninguna intervención humana. Los supuestos básicos utilizados para su creación y programación han sido considerarlos como agentes egoístas que compiten entre sí para maximizar sus ganancias y tratar de esquilmar a otros. Si las máquinas actúan de esa forma, cualquier ser humano que pretenda operar exitosamente en estos mercados debe convertirse también en otro agente egoísta para no perder sus inversiones y termina actuando de la misma manera que las máquinas, y por esta vía los humanos se convierten en un espejo de las máquinas especulativas y nuevamente terminan mecanizándose para maximizar sus ganancias sin sonrojarse y sin remordimientos éticos (Schirrmacher, 2014). El egoísmo absoluto de los agentes que era un postulado económico se transforma en un hecho al ser incorporado en las computadoras y adquiere el carácter de una realidad tangible por encima de sus creadores.

Otra aplicación de la informática ha sido intentar controlar a las personas que navegan en Internet al colocarlas en una burbuja informativa (verdadera o incluso falsa) para dirigir su conducta. Para ello primero realizan un perfil del individuo y luego tratan de «encerrarlo» con información coincidente dirigida a explotar sus vulnerabilidades para modificar su conducta. Este mecanismo se ha empleado para fines comerciales o políticos y parece haber influido en la polarización social que permitió la victoria de Donald Trump o del Brexit inglés. Estos procedimientos transforman nuevamente a las personas en una prolongación de las máquinas, aunque las víctimas perversamente puedan pensar que son decisiones que han tomado «libremente» con base en información que han visto en la red (Redacción BBC, 2018).

\section{La crisis actual}

Al concluir la Guerra Fría con la caída del muro de Berlín y el colapso de la URSS, desapareció la competencia militar y política entre dos formas de administrar el mecanicismo social. Esto se interpretó como un triunfo del modelo capitalista norteamericano sobre el socialista, y dado que disminuyó la competencia entre bloques de naciones se limitaron las inversiones en ciencia y tecnología destinadas a mantener 
el equilibrio militar y con ello se redujo la innovación y se estancó la mejora de la productividad. Asimismo, con el fin de incrementar las ganancias se procedió a reducir los beneficios sociales a la clase media desmantelando el estado de bienestar, pues ya no era necesario mantener la lealtad de la población nacional y se procedió a reducir los niveles de vida mediante la exportación de trabajo a los países menos desarrollados en donde había salarios más bajos y falta de protección social y del medio ambiente, con lo cual los trabajadores de los países desarrollados tuvieron que competir contra los bajos salarios y sus ingresos se deterioraron.

Las grandes corporaciones aseguraron el crecimiento de sus ingresos controlando a los reguladores, innovando las formas de evadir la ley, promoviendo la reducción de los impuestos a las grandes corporaciones. Decidieron controlar a los políticos para cambiar las leyes a su favor y evadieron responsabilidades, como se hizo evidente cuando la desregulación abrió la puerta de la crisis financiera y bancaria del 2008. Se privilegió una economía de corte rentista, y la transferencia de recursos hacia los que más tienen se aceleró, favoreciendo la desigualdad, la exclusión y el enojo social, debilitando a la democracia (Stiglitz, 2019).

Las elites gobernantes priorizaron sus ganancias y postergaron tomar acciones decisivas para incrementar la productividad de manera sostenible y amigable con el entorno, desfinanciaron la ciencia y el desarrollo tecnológico y redujeron la productividad y obstaculizaron la democracia para impedir ser regulados. No fueron capaces de impulsar una economía del conocimiento que mejorase la productividad y distribuyese los beneficios sociales.

En cambio, intentaron ganar tiempo aplicando los métodos de gobierno probados en los países latinoamericanos por todo tipo de demagogos. Los grupos beneficiarios de estas políticas transfieren fuertes recursos económicos para financiar campañas políticas, mediante las cuales manipulan a las multitudes con menor preparación poseedoras de una personalidad dirigida, promoviendo el racismo, la manipulación de emociones, las técnicas de control psicológico del tipo de Cambridge Analytica, y utilizan fake news, campañas de odio, y rumores, para inventar narraciones creíbles por los grandes grupos enojados por el deterioro de su nivel de vida; de esta forma se logró que estos grupos votasen por políticos comprometidos con los intereses corporativos para realizar acciones que seguirán beneficiando a los grupos económicos más poderosos a la vez que deterioran más la democracia e impulsan los conflictos raciales, la violencia y la represión, y el deterioro ecológico. A tal grado que la revista británica The Economist calificó a Donald Trump como un peronista en la Casa Blanca (Bello, 2017). 
Ello ha terminado por debilitar a los EE.UU. a nivel internacional, pues se ha retraído y ha sido incapaz de apoyar militarmente a sus aliados, y ha debilitado su presencia en el comercio global, colocando a los EE.UU. en una posición defensiva frente al gigante emergente de China, que tampoco es una democracia pero que ha sabido generar un proceso de industrialización acelerada y se ha movido junto con Rusia para ocupar el vacío que ha dejado Norteamérica. Ello ha creado nuevamente un clima similar al de la Guerra Fría y ha hecho reaccionar a algunos grupos de la elite norteamericana que pretenden retomar el control del país intentando reconstruir una economía deteriorada por la política de ganancias por encima de las personas y los países. No sabemos si lograrán superar la polarización social, reconstruir las redes del estado de bienestar y la protección social, detener el racismo, ganar la confianza de la población, impulsar la ciencia y la tecnología, incrementar la productividad y fortalecer su presencia militar, aunque tal vez sea demasiado tarde para recuperar lo perdido, pero en todo caso ello mostrará que la máxima de Milton Friedman, relativa a privilegiar las ganancias, destruye naciones enteras y que un país no puede reducirse a la mera suma de intereses corporativos, o para decirlo con mayor claridad: un Estado nación sinérgico y con propósitos democráticos claros puede explicar el surgimiento de grandes corporaciones, pero no puede reducirse a los intereses de las grandes corporaciones porque estas inmensas máquinas de obtener ganancias lo destruirán, pero también pueden destruir al planeta entero involucrándolo en una inmensa crisis ecológica y social.

La crisis de la Unión Soviética primero y ahora la crisis de los EE.UU. representan la crisis del mecanicismo social en su conjunto y con ello de la modernidad y de la sociedad derivada del reduccionismo newtoniano-laplaciano. Han creado una sociedad maquinal incapaz de gobernarse a sí misma a menos que se transforme y construyamos una sociedad más humana y sustentada en el paradigma de la biología y la ecología.

Debemos hacer notar que esta crisis es una crisis bioética, porque la obtención de ganancias a toda costa puso los intereses de las corporaciones por arriba de las personas y la ecología y la superación de la crisis actual demanda poner en el centro a la vida misma, porque solo la articulación de la vida y la ética puede darnos un puente al futuro para superar esta sociedad maquinal. 


\section{Recuperando la creatividad humana y de la vida}

La automatización de procesos, los sistemas de fabricación flexible, la robótica y la inteligencia artificial pueden permitir eliminar la necesidad del trabajo inhumano, rutinario y degradante, y al mismo tiempo incrementar la producción y el bienestar. El reto consiste en lograr que la desaparición de trabajos de baja calificación se transforme en la apertura de trabajos de alta calificación, relacionados con la investigación, el desarrollo y la innovación, y entre ambos tipos de trabajo median unos 10 o 15 años de estudio. Esta transición implica reducir la cantidad de personas sin iniciativa acostumbradas a seguir instrucciones de manera acrítica, a fin de formar ciudadanos empoderados. Para ello deberemos estimular y becar a decenas de millones de niños y jóvenes y a sus familias, educándolos a todos, para desarrollar un gran esfuerzo dirigido a transformar a los sectores obreros y a los marginados en profesionales, investigadores, diseñadores, educadores, biólogos y ecólogos, entre otros. El cambio implicará el tránsito del poder desde los pocos que controlan la producción hacia quienes produzcan conocimiento y nos permitan interactuar exitosamente con nuestro entorno para hacernos responsables de desarrollar la biósfera; volveremos más adelante sobre este punto.

La superación definitiva de la modernidad y el mecanicismo social requiere de una transferencia de poder, desde quienes son propietarios de las máquinas y el capital especulativo, hacia quienes generan innovaciones que permiten el desarrollo humano y de la biósfera. La parte creativa y humana debe gobernar a la parte maquinal y rutinaria que deviene en inconsciente y destructiva. Ello implica crear ambientes propicios para la generación y exploración de ideas nuevas y requiere de la libertad creadora y de la construcción de consensos; también demanda recuperar la democracia y lograr que los gobernados controlen a los gobernantes y que estos se abstengan de instrumentar manipulaciones en sinergia con las grandes corporaciones. El futuro será bioético o no será. Los mercados son indispensables para el desarrollo humano, pero deben responder también a un marco de responsabilidad social y favorecer el desarrollo de la biósfera. Hoy es posible utilizar la informática y los «big data» para mapear el desarrollo de la economía, el desarrollo humano y los ciclos biogeoquímicos de la biósfera, gravando con impuestos a quienes afecten a las personas, a la sociedad o a los ecosistemas.

Se ha acusado a la sociedad actual de ser antropocéntrica, pero ello es una falsa conciencia. La sociedad moderna es esencialmente mecanocéntrica y reduccionista y ha convertido a la mayoría de las personas en seres rutinarios y sin iniciativa, 
transformándolos en entes al servicio de un mecanismo productivo y social lineal que termina deteriorando la salud y la biósfera. Al perder nuestra variabilidad y creatividad nos hemos deshumanizado todos. Si pusiéramos a los seres humanos en el centro veríamos que somos un microcosmos de autoorganización, cooperación y creatividad y podríamos superar la crisis ecológica. No podremos ser plenamente humanos sin visualizar la vida como negatoentropía, evolución y desarrollo constante. Hemos colocado a la física y en especial a la mecánica en el centro; ha llegado el momento de colocar a la biología como ciencia que permite entender y ordenar al resto para abrir nuevas vías para el desarrollo humano y a las humanidades que nos hacen identificar lo valioso. Es la recuperación de la centralidad de lo biológico y de lo humano la que nos permitirá abrir nuevos caminos.

Entender los procesos de autoorganización en la ecología de la selva tropical, sus flujos de energía y ciclos biogeoquímicos es indispensable para aprender de ellos y generar una economía de lo viviente y autoorganizado contrapuesta a la linealidad mecánica. Así como los árboles y sus frutos son indispensables para la vida de los monos, los monos resultan indispensables para la vida de los árboles al propagar sus semillas y fertilizarlas generando mecanismos de sinergia que acrecientan la autoorganización de los ecosistemas. La prueba de que los seres humanos no son necesariamente destructores de la naturaleza la encontramos en algunos pueblos originarios que entienden que la selva posee un «espíritu» del cual hay que aprender. Esto implica reconocer que posee un sentido, autoorganización y dinámica propia, que puede ser comprendida para poder interactuar con ella de manera coherente y respetuosa, entendiendo su dinámica compleja y no lineal. El denominado antropocentrismo no es inherente a los seres humanos, sino producto de la visión lineal y mecánica dirigida a lograr efectos inmediatos, la cual nos ha generado una falsa conciencia de nosotros y de la naturaleza. Esta visión primitiva y mecánica ha terminado por eliminar nuestra humanidad y capacidad cooperativa y nos ha impedido ver el sentido de la vida como evolución y autodesarrollo.

Es necesario ir más allá del conservacionismo, que finalmente es una reacción newtoniana a la fuerza destructora de la sociedad mecánica, porque considera, de acuerdo con la tercera ley de Newton, que «a toda acción debemos oponer una reacción de sentido contrario e igual intensidad» para que el sistema se mantenga estable. Pero en la medida en que podemos utilizar nuestra capacidad intelectual para entender la dinámica de la biósfera, e identificar los fenómenos emergentes que nos sorprenden y demuestran que la vida es un sistema dinámico complejo, y que nosotros mismos somos producto de la evolución y la negatoentropía, la conciencia de que 
somos producto de la asimetría de la vida y que nosotros podemos catalizar procesos asimétricos negatoentrópicos que expanden el desarrollo de la biósfera, impulsaríamos los procesos de autoorganización que incrementan la biomasa y favorecen interacciones complejas que fortalecen los ciclos biogeoquímicos que dan sustento a la biósfera. Así podríamos arribar al ideal de Vladimir Vernadsky (Vernadsky, 1944) de construir una noósfera o biósfera bajo el desarrollo consciente de la humanidad, lo cual nos permitiría co-evolucionar. No podemos construir lo nuevo sin estudiar a las selvas y los ecosistemas más desarrollados. No podemos convertir a la biósfera en una fábrica, sino a la fábrica en un ser vivo dinámico adaptativo armónico con su entorno.

Así como el movimiento ecológico ha sabido poner en el centro a los problemas ambientales, hoy también debemos poner en el centro a la bioética como elemento articulador para dar valor al futuro de la vida y a los seres humanos. El futuro será bioético o no será.

\section{Conflicto de intereses}

El autor declara no tener ningún conflicto de intereses.

\section{Referencias}

Arendt, H. (1994). Los orígenes del totalitarismo 1. Barcelona: Planeta-Agostini.

Bello. (16 febrero 2017). A Peronist on the Potomac: Donald Trump through Latin American eyes. The Economist. Disponible en: https://www.economist.com/the-americas/2017/02/16/a-peronist-on-the-potomac

Chokshi, N. (16 septiembre 2020). House Report Condemns Boeing and F.A.A. in 737 Max Disasters. The New York Times. Disponible en: https://www.nytimes.com/2020/09/16/business/boeing-737-max-housereport.html?campaign_id=60\&emc=edit_na_20200916\&instance_id=0\&nl=breaking-news\&ref=cta \&regi_id=140325095\&segment_id=38230\&user_id= 0f1c868f33b457018e4cd53b27bcf88a

Coriat, B. (2008). El Taller y el cronómetro: ensayo sobre el taylorismo, el fordismo y la producción en masa. México: Siglo XXI.

Friedman, M. (13 septiembre 1970). A Friedman doctrine - The social responsibility of business is to increase its profits. New York Times Magazine, pp. 32-33.

Merkle, J. (1980). The Taylor System in Soviet Socialism. In Management and Ideology: The Legaacy of International Scientific Management Movement. pp. 103-133. Berkley: University of California.

Polanyi, M. (1968). Life's irreducible structure. Science, 160(3834), 1308-1312.

Redacción BBC. (2018). 5 claves para entender el escándalo de Cambridge Analytica que hizo que Facebook perdiera US\$37.000 millones en un día. BBC News [consultado 16 Sep 2020]. Disponible en: https://www.bbc.com/mundo/noticias-43472797

Riesman, D., Glazer, N. y Denney, R. (2001). The lonely crowd: a study of the changing American character. New Haven, CT: Yale University Press. 
Rifkin, J. (2010). La civilización empática: la carrera hacia una conciencia global en un mundo en crisis. Barcelona: Paidós Ibérica.

Schirrmacher, F. (2014). Ego: Las trampas del juego capitalista. Barcelona: Ariel.

Schrödinger, E. y Penrose, R. (1992). What is life? In What is life?: With mind and matter and autobiographical sketches. Cambridge: Cambridge University Press.

Stiglitz, J. (2019). People, power and profits: progresive capitalism for an age of discontent. New York: W.W. Norton \& Co,

Vernadsky, V. (1944). Problems in Biogeochemistry II: On the fundamental material-energetic distinction between living and no living natural bodies of the Biosphere. Transactions of the Connecticut Academy of Arts and Sciences.

Vetlesen, A. J. (2012). Technology, nature and ethics. En R. Bhaskar, K. G. Høyer, y P. Næss (Eds.), Ecophilosophy in a world of crisis: Critical realism and the Nordic contributions (pp. 25-43). London: Routledge. 